characteristics are as follows: onset in the first two decades, progressive course, extrapyramidal signs (dystonia, rigidity, or choreoathetosis), MRI hypodensities corresponding with necrosis and iron deposits in basal ganglia, especially globus pallidus and substantia nigra, and the "eye-of-the-tiger" sign (higher signal intensity in centre and low signal on each side of globus pallidus).

Corrobarative signs (at least two) include: spasticity, intellectual deterioration, retinitis pigmentosa and/or optic atrophy, positive family history (autosomal recessive), and sea-blue histiocytes in bone marrow and/or abnormal cytosomes in circulating lymphocytes. Disorders to be excluded include: Wilson's disease, neuronal ceroid lipofuscinosis, juvenile Huntington's disease, GM1galactosidase or hexosminidase A deficiencies. The differential diagnosis also includes the HARP syndrome, a possible phenotype of HSS (hypobetalipoproteinemia, acanthocytosis, retinitis pigmentosa, and pallidal degeneration). The pathology. including iron metabolism and its accumulation in the basal ganglia, and symptomatic therapy are also discussed. (Swaiman KF. Hallervorden-Spatz syndrome. Pediatr Neurol August 2001;25:102-108). (Respond: Dr Swaiman, 1821 University Avenue W, N-188, St Paul, MN 55104).

COMMENT. The rare association of acanthocytosis and HSS has been reported by Swisher CN et al (1972), and the familial late infantile HSS has been described as a form of neuroacanthocytosis (Malandrini A et al, 1996). The above issue of Pediatric Neurology also includes reports on "Iron in the HSS," (Koeppen AH, Dickson AC, 2001), "Iron overload, oxidative stress, and axonal dystrophy in brain disorders," (Chiuch CC, 2001), "Systemic iron metabolism: a review and implications for brain iron metabolism," (Rouault TA, 2001); "Iron and iron management proteins in neurobiology," (Connor JR et al, 2001), and "Basal ganglia motor function in relation to Hallervorden-Spatz syndrome," (Mink JW, 2001). It is postulated that dystonia and other extrapyramidal signs of HSS are attributed to degeneration of basal ganglia output neurons. (See Progress in Pediatric Neurology III, PNB Publ, 1997;pp560-565, for further references to Harp and HSS syndromes).

\title{
X-LINKED CHARCOT-MARIE-TOOTH DISEASE IN 93 PATIENTS
}

The clinical, electrophysiological and genetic features of 93 patients (41 males, 52 females) from 37 unrelated families with X-linked dominant CharcotMarie-Tooth (CMTX) disease are reported from the Hopital de la Salpetriere, Paris, France. Age at onset was younger in males than females: $15.4+/-9.6$ yrs vs $18.7+1-$ $13.1 \mathrm{yrs}$. The respective age ranges of onset were 1-40 yrs in males and 1-56 yrs in females $(\mathrm{P}=0.22)$. Onset before age 10 years occurred in $27 \%$ of males and $15 \%$ of females. Disease duration at time of examination was shorter in males than females: $18.3+/-14.6$ yrs in males and $23.9+/-13.7$ yrs in females $(\mathrm{P}=0.11)$. Males were more severely affected than females and had higher functional disability scores; they had more frequent muscle weakness, amyotrophy, proprioception loss, upper limb areflexia, and pes cavus. Females were more frequently asymptomatic than males (29\% vs $11 \%)$. Motor nerve conduction velocities, distal motor latencies, and compound muscle action potentials were slower, longer and lower, respectively, in males compared to females. In molecular genetic testing, 27 different CX32 mutations and one entire deletion of the CX32 coding sequence were present in the 37 families. Patients with age at onset before 10 years presented nonfunctional mutations, and the type of mutation influences the phenotype. (Dubourg O, Tardieu S, Birouk $\mathrm{N}$ et al. Clinical, electrophysiological and molecular genetic characteristics of 93 patients with X-linked Charcot-MarieTooth disease. Brain October 2001;124:1958-1967). (Respond: Dr O Dubourg, Inserm 
U289, Hopital de la Salpetriere, 47 boulevard de l'Hopital, 75651 Paris Cedex 13, France).

COMMENT. In this large series of patients with CMTX, differences between male and female patients are more apparent than in previous studies. Males are more severely affected than females, and onset before 10 years is also more frequent in males than females, occurring in $27 \%$ and $15 \%$ of patients, respectively. Patients with early onset present nonfunctional genetic mutations. These gender differences are also reflected in electrophysiological findings.

\section{EEG IN LATE INFANTILE NEURONAL CEROID LIPOFUSCINOSIS}

The clinical and electroencephalographic findings in 18 cases ( 8 girls, 10 boys) with late infantile neuronal ceroid lipofuscinosis (NCL) are reported from the University of Genova, and University of Siena, Italy. Age at onset ranged from 1 year 6 months to 6 years 6 months. Presenting symptoms were seizures in 12 patients, ataxia in 4 , and psychomotor regression followed by seizures in 2 . All patients had developed a progressive myoclonic epilepsy at foilow-up. Neuroimaging in $12 / 18$ patients showed cerebral atrophy in 6 and cerebellar atrophy in 4 . Electron microscopy of skin, conjunctiva or rectal biopsies showed characteristic curvilinear bodies and fingerprint profiles.

EEG findings confirmed the classical and diagnostic pseudoperiodic atypical high voltage slow spike and wave pattern in $15 / 18$ cases, as previously reported, and some additional variants are described. While awake, background activity is slowed and pseudoperiodic discharges are at intervals of 8-10 sec. The response to eye opening and closing is absent, and the periodic pattern remains unmodified. During sleep, there is an absence of sleep spindles, and the periodic discharges are less evident. Treatment with ACTH partially controlled seizures but failed to block the periodic pattern. (Veneselli E, Biancheri R, Buoni S, Fois A. Clinical and EEG findings in 18 cases of late infantile neuronal ceroid lipofuscinosis. Brain Dev August 2001;23:306-311). (Respond: Dr Edvige Veneselli, Child Neuropsychiatry Unit, G Gaslini Institute, University of Genova, Largo G Gaslini 5, 16147 Genova, Italy).

COMMENT. The value of the EEG in the early diagnosis of late infantile neuronal ceroid lipofuscinosis is confirmed by this study of 18 cases. The first report of the characteristic pseudoperiodic pattern found in cases of LINCL was that of Pampiglioni G and Harden A in 1977, at Great Ormond Street Children's Hospital, London, UK. Subsequent reports of the EEG in LINCL and other types of NCL from the same center include those of Boyd SG and Harden A (1991), and Williams RE, Boyd SG, lake BD, and Wilson J (1997). Recently, molecular genetic analyses have clarified the diagnosis in some NCL variants.

The classification of various forms of neuronal ceroid lipofuscinosis is described by Dyken PR (In Progress in Pediatric Neurology II. PNB Publishers, 1994;481-485). These include: Infantile (Santavuori), Late Infantile (Bielschowsky), Early Juvenile (Zeman-Dyken), Juvenile (Batten), Adult Recessive (Kufs), Adult Dominant (Boehme), and Atypical (Dyken-Wisniewski). Fifteen atypical forms of NCL are identified, and 21 subtypes, most representing separate diseases with different chromosome defects: chromosome 1 defect in the infantile form; 13 in early juvenile, 16 in the juvenile form, and neither chromosome 1,13 , nor 16 in the LINCL. (Dyken, 1994). 